\title{
SARS-CoV-2 Infection during Pregnancy in a Rural Midwest All-delivery Cohort and Associated Maternal and Neonatal Outcomes
}

\author{
Haley A. Steffen, BA ${ }^{1, *}$ Samantha R. Swartz, BS ${ }^{1, *} \quad$ J. Brooks Jackson, MD, MBA ${ }^{2}$ \\ Kimberly A. Kenne, MD, MCR ${ }^{3}$ Patrick P. Ten Eyck, MS, PhD ${ }^{4}$ Abbey S. Merryman, MD ${ }^{3}$ \\ Claire N. Castaneda, BSE ${ }^{1}$ Katharine Marsden, BA ${ }^{1}$ Timothy Maxwell, BS ${ }^{1}$ Anna E. Merrill, PhD $^{2}$ \\ Matthew D. Krasowski, MD, PhD ${ }^{2}$ Mary B. Rysavy, MD ${ }^{30}$
}

1 University of lowa Carver College of Medicine, lowa City, lowa

${ }^{2}$ Department of Pathology, University of lowa, lowa City, lowa

${ }^{3}$ Department of Obstetrics and Gynecology, University of lowa, lowa City, lowa

${ }^{4}$ Institute for Clinical and Translational Science, University of lowa, lowa City, lowa

Am J Perinatol 2021;38:614-621.
Address for correspondence Mary B. Rysavy, MD, Department of Obstetrics and Gynecology, PFP 51235, 200 Hawkins Drive, lowa City, IA 52242 (e-mail: mary-rysavy@uiowa.edu).

\begin{abstract}
Keywords

- COVID-19

- pregnancy

- racial disparities

- SARS-CoV-2

- seroprevalence

Objective This study aimed to estimate the prevalence of severe acute respiratory syndrome-coronavirus-2 (SARS-CoV-2) among pregnant patients at the time of delivery in a rural Midwest tertiary care hospital and to examine demographics, clinical factors, and maternal and neonatal outcomes associated with SARS-CoV-2 infection during pregnancy.

Study Design This prospective cohort study included all delivering patients between May 1 and September 22, 2020 at the University of lowa Hospitals and Clinics. Plasma SARS-CoV-2 antibody testing was performed. SARS-CoV-2 viral reverse-transcription polymerase chain reaction (RT-PCR) results and maternal and neonatal outcomes were collected from the electronic medical record. Data were analyzed using univariate statistical methods with clustering for multiple births.

Results In total, 1,000 patients delivered between May 1 and September 22, 2020. Fiftyeight (5.8\%) were SARS-CoV-2 antibody positive. Twenty-three also tested viral positive during pregnancy. Three of $1,000(0.3 \%)$ were viral positive on admission but antibody negative. The median age was 30 years (interquartile range [IQR]: 26-33 years) and body mass index was $31.75 \mathrm{~kg} / \mathrm{m}^{2}$ (IQR $27.7-37.5 \mathrm{~kg} / \mathrm{m}^{2}$ ). The cesarean delivery rate was $34.0 \%$. The study population was primarily white (71.6\%); however, $41.0 \%$ of SARS-CoV- 2 infected patients identified as Black, $18.0 \%$ as Hispanic/Latino, $3.3 \%$ as Native Hawaiian/Pacific Islander, and only $27.9 \%$ as White $(p<0.0001)$. SARS-CoV-2 infection was more likely in patients without private insurance $(p=0.0243)$. Adverse maternal and/or neonatal outcomes were not more likely in patients with evidence of infection during pregnancy. Two SARS-CoV-2 infected patients were admitted to the intensive care unit. There were no maternal deaths during the study period.
\end{abstract}

received

November 30, 2020

accepted after revision

January 9, 2021

published online

February 21, 2021 (c) 2021. Thieme. All rights reserved.

Thieme Medical Publishers, Inc.,

333 Seventh Avenue, 18th Floor,

New York, NY 10001, USA
DOI https://doi.org/

$10.1055 / \mathrm{s}-0041-1723938$.

ISSN 0735-1631. 
Conclusion In this largely rural Midwest population, $6.1 \%$ of delivering patients had evidence of past or current SARS-CoV-2 infection. Rates of SARS-CoV-2 during pregnancy were higher among racial and ethnic minorities and patients without private insurance. The SARS-CoV-2 infected patients and their neonates were not found to be at increased risk for adverse outcomes.

\section{Key Points}

- SARS-CoV-2 seroprevalence rate in pregnant population in Iowa is $5.8 \%$.

- Infections are higher among minorities, non-English speakers, and patients without private insurance.

- No increased adverse maternal/neonatal outcomes observed for SARS-CoV-2 infected mothers.

First appearing in Wuhan, China, in December of 2019, severe acute respiratory syndrome-coronavirus-2 (SARS-CoV-2) is a novel respiratory virus that has caused a worldwide pandemic. Although initially more rampant in urban areas, the virus has affected urban and rural areas alike across the United States. In Iowa, the first cases of SARS-CoV-2/novel coronavirus disease 2019 (COVID-19) were diagnosed in March 2020, but cases have continued to accumulate with 1 in 24 Iowans testing positive for the virus as of this writing. ${ }^{1}$ While the literature on COVID-19 has expanded rapidly in recent months, no studies have yet reported on COVID-19 in pregnancy in a largely rural population; and few studies have reported on an all-delivery cohort to determine an accurate COVID-19 antibody seroprevalence in a pregnant population.

It remains unclear how infection with SARS-CoV-2 in pregnancy is related to maternal and neonatal outcomes. Previous reports regarding other coronaviruses, such as SARS-CoV and Middle East respiratory syndrome coronavirus (MERS-CoV), showed potential for poor obstetric and perinatal outcomes when women were infected during pregnancy, ${ }^{2}$ with maternal hypoxia as a possible mechanism. ${ }^{3}$ Reports to date of COVID-19 in pregnancy have described maternal respiratory symptoms, including severe respiratory distress, similar to the nonpregnant population. Research suggests that hospital and intensive care unit (ICU) admission rates are higher in pregnant patients with COVID-19 than nonpregnant patients of reproductive age with COVID-19. ${ }^{4}$ Current limited literature also suggests possible associations between COVID-19 infection in pregnancy and adverse outcomes including fetal distress, premature rupture of membranes, preterm delivery, preeclampsia, and fetal growth restriction. ${ }^{5-7}$

When looking at which factors may affect who becomes infected with SARS-CoV-2, it has become clear that demographic factors, such as race and socioeconomic status, play a large role. ${ }^{8}$ Preexisting health conditions and chronic disease have also been linked to increased infection rates and disease complications. ${ }^{9}$ Multiple studies have suggested that women with asthma and obesity are more likely to have severe COVID-19 in pregnancy. ${ }^{10,11}$

This study aimed to estimate the prevalence of SARS-CoV2 infection among pregnant patients at the time of delivery at the University of Iowa Hospitals and Clinics (UIHC) between
May 1 and September 22, 2020. We sought to assess which demographic or clinical factors may be associated with increased risk of infection in our population, as well as to describe any maternal and/or neonatal adverse outcomes associated with SARS-CoV-2 infection during pregnancy.

\section{Materials and Methods}

This prospective cohort study included all pregnant patients who delivered at UIHC between May 1 and September 22, 2020. SARS-CoV-2 reverse-transcription polymerase chain reaction (RT-PCR) testing was performed on delivery admission for all patients as part of routine hospital care starting on April 9, 2020. Excess plasma from routinely collected blood samples was used to determine the seroprevalence of SARS-CoV-2 antibody within this population. Given the first confirmed case of COVID-19 in Iowa was documented on March 8, 2020, antibody positive patients in this study period would most likely have been infected during their current gestation. The institutional review board at the University of Iowa approved this study (institutional review board [IRB] ID no.: 202004278). The project was internally funded.

Residual EDTA plasma from patient samples was tested for antibody presence using the LIAISON SARS-CoV-2 S1/S2 immunoglobulin (Ig)-G (DiaSorin) and the Elecsys AntiSARS-CoV-2 (Roche) assays. Prior studies have shown that these assays become positive, at the earliest, 5 to 7 days after COVID-19 RT-PCR positivity and are nearly $100 \%$ positive by 3 weeks. ${ }^{12,13}$ Deidentified existing plasma samples from 103 healthy individuals collected at least 1 year prior to the COVID-19 outbreak were tested as negative controls. Discrepancies between the DiaSorin and Roche assays were resolved using a third assay: the EUROIMMUN Anti-SARSCoV-2 enzyme-linked immunosorbent assay (ELISA) IgG.

Demographic and clinical data were obtained from the electronic medical record and double entered in a Research Electronic Data Capture (REDCap) database. Demographic data included race and ethnicity which was self-reported during the hospital registration process. The racial categories used correspond with the National Institutes of Health (NIH)'s racial and ethnic categories. ${ }^{14}$ SARS-CoV-2 infection severity was categorized as follows: mild symptoms were 
defined as cases limited to upper respiratory congestion, headache, and/or gastrointestinal symptoms. Moderate-tosevere cases required documentation of one or more of the following: cough, sore throat, myalgia, chest pain, loss of taste/smell, dyspnea, and/or hypoxia. Summary statistics are provided for demographic, clinical, and delivery outcome measures stratified by SARS-CoV-2 infection status. Categorical variables are displayed as counts and percentages, while continuous variables are displayed as means (standard deviations) or medians (interquartile ranges [IQRs]), depending on the normality of each distribution. Tests for differences in outcome between the SARS-CoV-2-positive and -negative subsets were conducted using Fisher's exact, two-sample $t$ - or Wilcoxon rank sum tests. Further comparisons of neonatal outcome measures utilized the generalized linear mixed modeling (GLMM) framework to account for multiple births from some mothers and varying distributions (normal and binary) for the outcomes. This method allowed us to identify if the analyses were sensitive to adjusting for repeated measurements. Any comparison with a $p$-value of $<0.05$ was considered statistically significant. All analyses were conducted using SAS 9.4.

\section{Results}

A total of 1,000 pregnant patients delivered at UIHC between May 1 and September 22, 2020. Population characteristics are listed in -Table 1.

Fifty-eight of the women (5.8\%) were classified as having SARS-CoV-2 antibodies by both the DiaSorin IgG and Roche total antibody assays, with discrepancies resolved by the EUROIMMUN antibody assay. Only eight patients had discrepant results between the DiaSorin and Roche antibody assays. Four were positive by DiaSorin but negative by Roche and EUROIMMUN; these were classified as negative for COVID-19. Four were negative by DiaSorin and positive by Roche. Of these, one specimen was also positive by EUROIMMUN and was COVID-19 RT-PCR positive; this was classified as positive for COVID-19. The other three were negative by EUROIMMUN in addition to DiaSorin and were classified as negative for COVID-19. All 103 pre-COVID-19 samples were confirmed SARS-CoV-2 antibody negative.

Twenty-three women in the study tested viral positive by RT-PCR at some point during their pregnancy. Of these 23 women, 13 tested positive prior to their admission and 10 tested positive during routine admission screening for labor and delivery. Three of the patients who tested viral positive on admission were found to be antibody negative, suggesting that they were most likely infected with the virus but early in their infection course and had not yet formed antibodies to the virus. All three of these patients were asymptomatic (-Table 1). Thus, 61 delivering patients (6.1\%) had evidence of past or current SARS-CoV-2 infection as defined as viral or antibody positivity at some point during their pregnancy, referred to from this point on as COVID-19 positive (absence of SARS-CoV-2 infection is referred to as COVID-19 negative).

Of the 61 COVID-19-positive patients, 31 (50.8\%) remained asymptomatic during their ante- and intrapartum
Table 1 Description of study population

\begin{tabular}{|c|c|}
\hline Characteristic & $\begin{array}{l}\text { Population } \\
(n=1,000)\end{array}$ \\
\hline $\begin{array}{l}\text { Maternal age (y) } \\
\text { Median (IQR) }\end{array}$ & $30(26-33)$ \\
\hline $\begin{array}{l}\text { BMI }\left(\mathrm{kg} / \mathrm{m}^{2}\right) \\
\text { Median (IQR) }\end{array}$ & $31.7(27.7-37.5)$ \\
\hline $\begin{array}{l}\text { Gestational age at admission (wk) } \\
\text { Median (IQR) } \\
\text { Gestational age at delivery (wk) } \\
\text { Median (IQR) }\end{array}$ & $\begin{array}{l}39^{0 / 7}\left(37^{2 / 7}-39^{5 / 7}\right) \\
39^{0 / 7}\left(37^{3 / 7}-39^{5 / 7}\right)\end{array}$ \\
\hline \multicolumn{2}{|l|}{$\begin{array}{l}\text { Gestation } \\
n(\%)\end{array}$} \\
\hline Singleton & $965(96.5)$ \\
\hline Twin & $34(3.4)$ \\
\hline Triplet & $1(0.1)$ \\
\hline \multicolumn{2}{|l|}{$\begin{array}{l}\text { Mode of delivery } \\
n(\%)\end{array}$} \\
\hline Vaginal & $647(64.7)$ \\
\hline Assisted vaginal & $48(4.8)$ \\
\hline Cesarean & $340(34.0)$ \\
\hline SARS-CoV-2 antibody positive ${ }^{a}$ & $58(5.8)$ \\
\hline $\begin{array}{l}\text { SARS-CoV-2 RT-PCR positive } \\
\text { prior to admission }{ }^{\mathrm{b}}\end{array}$ & $13(1.3)$ \\
\hline $\begin{array}{l}\text { SARS-CoV-2 RT-PCR positive } \\
\text { on admission }{ }^{c}\end{array}$ & $10(1.0)$ \\
\hline $\begin{array}{l}\text { Evidence of past/current } \\
\text { SARS-CoV-2 infection }\end{array}$ & $61(6.1)$ \\
\hline Asymptomatic & $31(50.8)$ \\
\hline Mild symptoms & $12(19.7)$ \\
\hline Moderate-to-severe symptoms & $17(27.9)$ \\
\hline
\end{tabular}

Abbreviations: BMI, body mass index; IQR, interquartile range; RT-PCR, reverse-transcription polymerase chain reaction; SARS-CoV-2, severe acute respiratory syndrome-coronavirus- 2 .

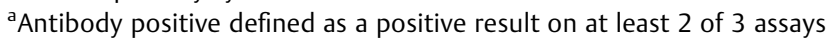
including DiaSorin, Roche, or EUROIMMUN.

${ }^{b}$ All patients RT-PCR prior to admission were also antibody positive.

'Seven of ten patients RT-PCR positive on admission were also antibody positive.

periods. Then, $19.7 \%$ of patients reported mild symptoms, whereas $27.9 \%$ reported moderate-to-severe symptoms. Two (3.3\%) of the 61 COVID-19-positive patients required ICU admission. One of the patients received treatment with convalescent plasma and a nasal cannula for advanced respiratory support. The second patient did not receive treatment with convalescent plasma, remdesivir, or hydroxychloroquine. However, she did receive advanced respiratory support including extracorporeal membrane oxygenation (ECMO). ${ }^{15}$

Collected study population characteristics according to SARS-CoV-2 infection status are listed in -Table 2. Despite nearly $92 \%$ of the study population identifying English as their preferred language, almost half (47.5\%) of the COVID-19positive patients identified a preferred language other than English. Similarly, nearly $72 \%$ of the study population self- 
identified as White, yet a majority of COVID-19-positive patients were Black or Hispanic/Latino (59.0\%). Also, 52.5\% of COVID-19-positive patients had public insurance and $8.2 \%$ had no insurance, while only $39.3 \%$ had private insurance $(p=0.0243)$, indicating significantly different rates of COVID-19 infection between insurance groups. Patients who had both private and public insurance were classified with the public insurance group. Nearly all (99.8\%) of delivering mothers had access to at least some prenatal care. Any outpatient clinic visits prior to delivery admission were counted as prenatal care, including visits at the local free antenatal clinic for uninsured patients. No other antepartum clinical factors were found to be associated with SARS-CoV-2 infection, including body mass index (BMI), diabetes, hypertensive disease, asthma, or HIV.

Maternal outcomes according to SARS-CoV-2 infection status are listed in -Table 3. No significant associations were seen between having past or current SARS-CoV-2 infection and maternal adverse outcomes. COVID-19-positive patients did not have a significantly increased length of stay. In the study population, 23\% of COVID-19-positive patients experienced postpartum hemorrhage versus $16.3 \%$ of COVID-19-negative patients $(p=0.213)$. Postpartum hemorrhage is defined as blood loss of greater than $1 \mathrm{~L}$.

Neonatal outcomes according to maternal SARS-CoV-2 infection status are listed in -Table 4. There were also no significant associations between mothers' SARS-CoV-2 infection status and any of the studied neonatal outcomes. Of the 65 neonates born to mothers with evidence SARS-CoV-2 infection, 17 underwent SARS-CoV-2 RT-PCR testing. The neonatal testing was performed either because the mother had a symptomatic SARS-CoV-2 infection or as part of routine asymptomatic testing in the neonatal ICU (NICU). All neonates tested were negative, but 48 neonates were not tested during their newborn hospital stay. Overall, there was no evidence of neonatal SARSCoV-2 infection. Neonates with four or more separate malformations or with chromosomal abnormalities were categorized as having a genetic/syndromic malformation. Of the 65 babies born to COVID-19 positive mothers, 10 (15.4\%) had malformations, compared to 117 (12.1\%) of 971 babies born to COVID-19 negative mothers $(p=0.413)$. Three of $65(4.6 \%)$ babies born to COVID-19-positive mothers and 12 of 971 (1.2\%) of babies born to COVID-19-negative mothers were not born alive. In the COVID-19-positive population, these were due to intrauterine fetal demise (IUFD) at 22 weeks, IUFD at 26 weeks, and labor induction at 21 weeks due to multiple fetal anomalies. In the COVID-19-negative population, these situations included termination of pregnancy by dilation and evacuation or induction of labor for cases that included IUFD or fetal anencephaly. There were 10 total neonatal deaths within 28 days of life (1.0\%), and all these infants were born to mothers who were COVID-19 negative.

\section{Discussion}

\section{Principal Findings}

At our Midwest tertiary care hospital from May 1 to September $22,2020,6.1 \%$ of delivering patients had evidence of past or current SARS-CoV-2 infection. The seroprevalence of SARSCoV-2 antibodies in the population was $5.8 \%$. Overall, $50.8 \%$ of COVID+ patients in our study were completely asymptomatic. We did not find a higher rate of SARS-CoV-2 infection or worse outcomes among patients with higher BMI or with asthma. The number of SARS-CoV-2 infections in pregnant women at UIHC was disproportionately greater among racial and ethnic minorities as represented by self-identified primary language and race/ethnicity, as well as among patients without private insurance. There were no significant associations found between SARS-CoV-2 infection during pregnancy and adverse maternal or neonatal outcomes.

\section{Comment}

We found a SARS-CoV-2 seroprevalence of $5.8 \%$, which is lower than studies of more urban areas. A seroprevalence of $16.1 \%$ was identified in a recent study of New York women admitted to Labor and Delivery using the Roche assay. ${ }^{16}$ Another study of seroprevalence in three universities in Barcelona, Spain, from April 14 to May 5, 2020, found a seroprevalence of $14 \%$ using assays different from our study. ${ }^{17}$ As of September 22, the Iowa Department of Public Health reported a seroprevalence of $6 \%$ for the state of Iowa. ${ }^{18}$ Over $50 \%$ of COVID-19-positive patients in our study population had asymptomatic infections. A high asymptomatic infection rate has also been noted in other pregnant populations where RT-PCR viral testing of asymptomatic individuals was performed. A description of universal SARS-CoV-2 testing in an obstetric population in New York showed that $13 \%$ of women were positive for the virus by RT-PCR but had no symptoms. Only $1.9 \%$ of the patients screened positive and had symptoms. ${ }^{18}$ Similar findings were reported in a Chicago hospital in April, where 3.6\% of 635 women admitted for delivery were viral positive but $43 \%$ of these women were asymptomatic. ${ }^{19}$

Notably, the average BMI at delivery for our entire population was obese, with a median BMI of $31.7 \mathrm{~kg} / \mathrm{m}^{2}$. The lack of findings in our study for patients with obesity and asthma contrasts with prior studies from other institutions, but likely reflects the low disease severity overall in the population. The cesarean delivery rate at UIHC (34.0\%) for the study period is slightly above the 2018 national average of $31.9 \%,{ }^{20}$ likely reflecting our referral base of high-risk patients and the large number of patients with high BMI. The obesity of the general population in Iowa is approximately $35 \%{ }^{21}$ For reference, BMI changes due to pregnancy weight gain vary significantly but increases 0.5 to 10 points on average. ${ }^{22}$

While only $8.5 \%$ of our study patients were non-English speaking, this group accounts for $52.5 \%$ of COVID-19-positive cases. Also, 59.0\% of COVID-19-positive cases were in patients identified as Black or Hispanic/Latino ethnicity, although they only made up $20.5 \%$ of the total study population. These results support previous studies that suggest patients facing language barriers and/or members of racial and ethnic minorities are at higher risk of SARS-CoV-2 infection in the United States. A study analyzing confirmed COVID-19 cases at a Louisiana medical center from May 2020 


\begin{tabular}{|c|c|c|c|c|}
\hline Demographic/clinical characteristic & $\begin{array}{l}\text { Population } \\
n=1,000\end{array}$ & $\begin{array}{l}\text { COVID-19+ } \\
n=61\end{array}$ & $\begin{array}{l}\text { COVID-19- } \\
n=939\end{array}$ & $p$-Value ${ }^{a}$ \\
\hline $\begin{array}{l}\text { Maternal age }(\mathrm{y}) \\
\text { Median (IQR) }\end{array}$ & $30,26-33$ & $28,24-32$ & $30,26-33$ & 0.028 \\
\hline $\begin{array}{l}\text { BMI }\left(\mathrm{kg} / \mathrm{m}^{2}\right) \\
\text { Median (IQR) }\end{array}$ & $31.7(27.7-37.5)$ & $31.7(27.7-37.5)$ & $32.0(27.5-37.7)$ & 0.933 \\
\hline $\begin{array}{l}\text { Gestational age at admission (wk) } \\
\text { Median (IQR) }\end{array}$ & $39^{0 / 7}\left(37^{2 / 7}-39^{5 / 7}\right)$ & $38^{6 / 7}\left(37^{0 / 7}-39^{4 / 7}\right)$ & $39^{0 / 7}\left(37^{3 / 7}-39^{5 / 7}\right)$ & 0.231 \\
\hline $\begin{array}{l}\text { Gestational age at delivery (wk) } \\
\text { Median (IQR) }\end{array}$ & $39^{0 / 7}\left(37^{3 / 7}-39^{5 / 7}\right)$ & $39^{0 / 7}\left(37^{1 / 7}-39^{4 / 7}\right)$ & $39^{0 / 7}\left(37^{3 / 7}-39^{5 / 7}\right)$ & 0.272 \\
\hline $\begin{array}{l}\text { Diabetes (T1DM, T2DM, gestational) } \\
n(\%)\end{array}$ & $172(17.2)$ & $8(13.7)$ & $147(15.6)$ & 0.709 \\
\hline \multicolumn{5}{|l|}{ Antepartum/intrapartum hypertension } \\
\hline Chronic & $89(8.9)$ & $4(6.6)$ & $85(9.1)$ & 0.646 \\
\hline Gestational & $98(9.8)$ & $4(6.6)$ & $94(10)$ & 0.506 \\
\hline Preeclampsia without severe features & $38(3.8)$ & $3(4.9)$ & $35(3.7)$ & 0.500 \\
\hline Preeclampsia with severe features & $55(5.5)$ & $6(9.8)$ & $49(5.2)$ & 0.140 \\
\hline Eclampsia & $1(0.1)$ & 0 & $1(0.11)$ & $>0.999$ \\
\hline $\begin{array}{l}\text { Asthma } \\
n(\%)\end{array}$ & $133(13.3)$ & $4(6.6)$ & $129(13.7)$ & 0.122 \\
\hline $\begin{array}{l}\text { HIV } \\
n(\%)\end{array}$ & $2(0.2)$ & 0 & $2(0.2)$ & $>0.999$ \\
\hline \multicolumn{5}{|l|}{$\begin{array}{l}\text { Language } \\
n(\%)\end{array}$} \\
\hline English & $915(91.5)$ & $32(52.5)$ & $883(94.0)$ & \multirow[t]{9}{*}{$<0.001$} \\
\hline Spanish & $30(3.0)$ & $7(11.5)$ & $23(2.5)$ & \\
\hline French & $17(1.7)$ & $11(18.0)$ & $6(0.6)$ & \\
\hline Portuguese & $1(0.1)$ & 0 & $1(0.11)$ & \\
\hline Mandarin & $2(0.2)$ & 0 & $2(0.21)$ & \\
\hline Lingala & $2(0.2)$ & $1(1.6)$ & $1(0.1)$ & \\
\hline Arabic & $12(1.2)$ & $1(1.6)$ & $11(1.2)$ & \\
\hline Haka Chin & $2(0.2)$ & $2(3.3)$ & 0 & \\
\hline Other & 19 (1.9) & $7(11.5)$ & $12(1.2)$ & \\
\hline \multicolumn{5}{|l|}{$\begin{array}{l}\text { Race/ethnicity } \\
n(\%)\end{array}$} \\
\hline White & $716(71.6)$ & $17(27.9)$ & $699(74.4)$ & \multirow[t]{8}{*}{$<0.001$} \\
\hline African American/Black & $118(11.8)$ & $25(41.0)$ & $93(9.9)$ & \\
\hline Hispanic/Latino & $87(8.7)$ & $11(18.0)$ & $76(8.1)$ & \\
\hline American Indian/Alaska Native & $3(0.3)$ & $1(1.6)$ & $2(0.2)$ & \\
\hline Native Hawaiian/Pacific Islander & $4(0.4)$ & $2(3.3)$ & $2(0.2)$ & \\
\hline Asian & $45(4.5)$ & $4(6.6)$ & $41(4.4)$ & \\
\hline Multiracial/two or more races & 19 (1.9) & 0 & $19(2.0)$ & \\
\hline Declined to answer & $8(0.8)$ & $1(1.6)$ & $7(0.8)$ & \\
\hline \multicolumn{5}{|l|}{$\begin{array}{l}\text { Insurance type } \\
n(\%)\end{array}$} \\
\hline Private only & $513(51.3)$ & $24(39.3)$ & $489(52.1)$ & \multirow[t]{3}{*}{0.0243} \\
\hline Medicare/Medicaid & $456(45.6)$ & $32(52.5)$ & $424(45.2)$ & \\
\hline None & $31(3.1)$ & $5(8.2)$ & $26(2.8)$ & \\
\hline $\begin{array}{l}\text { Any prenatal care } \\
n(\%)\end{array}$ & $998(99.8)$ & $61(100)$ & $937(99.8)$ & $>0.999$ \\
\hline
\end{tabular}

Abbreviations: BMI, body mass index, COVID-19, novel coronavirus disease 2019; HIV, human immunodeficiency virus; IQR, interquartile range; SARS-CoV-2, severe acute respiratory syndrome-coronavirus-2; T1DM, type 1 diabetes mellitus, T2DM, type 2 diabetes mellitus.

'Wilcoxon's rank sum and Fisher's exact tests used to determine $p$-values. 


\begin{tabular}{|lllll|}
\hline Table 3 Maternal outcomes and SARS-CoV-2 infection status & & & \\
\hline Maternal Outcome & $\begin{array}{l}\text { Population } \\
\boldsymbol{n}=\mathbf{1 , 0 0 0}(\%)\end{array}$ & $\begin{array}{l}\text { COVID-19+ } \\
\boldsymbol{n}=\mathbf{6 1}(\%)\end{array}$ & $\begin{array}{l}\text { COVID-19- } \\
\boldsymbol{n}=\mathbf{9 3 9}(\%)\end{array}$ & p-Value \\
Premature rupture of membranes & $90(9.0)$ & $6(9.8)$ & $84(8.9)$ & 0.816 \\
Preterm labor & $77(7.7)$ & $3(4.9)$ & $74(7.9)$ & 0.618 \\
Antenatal hypertensive disease & $242(24.2)$ & $14(23.0)$ & $228(24.3)$ & 0.879 \\
Placental abruption & $21(2.1)$ & $1(1.6)$ & $20(2.1)$ & $>0.999$ \\
Chorioamnionitis & $32(5.2)$ & $2(6.3)$ & $30(5.2)$ & 0.680 \\
Nonreassuring fetal status during labor & $71(11.6)$ & $5(15.6)$ & $66(11.4)$ & 0.403 \\
Morbidly adherent placenta & $1(0.2)$ & 0 & $1(0.2)$ & $>0.999$ \\
Unscheduled cesarean & $206(20.6)$ & $15(24.6)$ & $191(20.3)$ & 0.416 \\
Pyelonephritis & $6(0.6)$ & $1(1.6)$ & $5(0.5)$ & 0.315 \\
Postpartum hemorrhage & $167(16.7)$ & $14(23)$ & $153(16.3)$ & 0.213 \\
Postpartum hypertensive disease during the delivery admission & $204(20.4)$ & $14(23)$ & $190(20.2)$ & 0.623 \\
Postpartum endometritis during the delivery admission & $9(0.9)$ & $2(3.3)$ & $7(0.7)$ & 0.100 \\
Postpartum preeclampsia & $85(8.5)$ & $8(13.1)$ & $77(8.2)$ & 0.230 \\
Intensive care unit admission & $6(0.6)$ & $2(3.3)$ & $4(0.4)$ & 0.047 \\
Prolonged postpartum stay & $30(3.0)$ & $4(6.6)$ & $26(2.8)$ & 0.104 \\
\hline
\end{tabular}

Abbreviations: COVID-19, novel coronavirus disease 2019; SARS-CoV-2, severe acute respiratory syndrome-coronavirus-2.

\begin{tabular}{|lllll|}
\hline \multicolumn{3}{|l}{ Table 4 Neonatal outcomes and maternal SARS-CoV-2 Infection Status } \\
\hline Neonatal Outcome & $\begin{array}{l}\text { Population } \\
\boldsymbol{n}=\mathbf{1 , 0 3 6}(\%)\end{array}$ & $\begin{array}{l}\text { Maternal COVID-19+ } \\
\boldsymbol{n}=\mathbf{6 5}(\%)\end{array}$ & $\begin{array}{l}\text { Maternal COVID-19- } \\
\boldsymbol{n}=\mathbf{9 7 1}(\%)\end{array}$ & $\boldsymbol{p}$-Value \\
Sepsis & $39(3.8)$ & $2(3.1)$ & $37(3.8)$ & $>0.999$ \\
Hypoglycemia & $264(25.5)$ & $13(20)$ & $251(25.9)$ & 0.377 \\
Malformations & $127(12.3)$ & $10(15.4)$ & $117(12.1)$ & 0.434 \\
Cardiac & $52(5)$ & $3(4.6)$ & $49(5.1)$ & $>0.999$ \\
Genitourinary & $28(2.7)$ & $3(4.6)$ & $25(2.6)$ & 0.413 \\
Neuro/brain & $9(0.9)$ & $1(1.5)$ & $8(0.8)$ & 0.443 \\
Head and neck & $8(0.8)$ & 0 & $8(0.8)$ & $>0.999$ \\
Gastrointestinal & $5(0.5)$ & $1(1.5)$ & $4(0.4)$ & 0.277 \\
Musculoskeletal & $20(1.9)$ & 0 & $20(2.1)$ & 0.631 \\
Genetic/syndromic & $24(2.3)$ & $4(6.2)$ & $20(2.1)$ & 0.058 \\
Absence of live birth & $15(1.4)$ & $3(4.6)$ & $12(1.2)$ & 0.062 \\
Neonatal death & $10(1)$ & 0 & $10(1)$ & $>0.999$ \\
\hline
\end{tabular}

Abbreviations: COVID-19, novel coronavirus disease 2019; SARS-CoV-2, severe acute respiratory syndrome-coronavirus-2.

found that $76.9 \%$ of patients hospitalized for COVID-19 and $70.6 \%$ of those who died from COVID-19 were Black, although Black patients only accounted for $30.9 \%$ of their patient population. ${ }^{23}$ In July 2020, a meta-analysis by Khalil et al reported that $50.8 \%$ of all pregnant COVID-19 infected patients were Black, Asian, or of another ethnic minority group. ${ }^{24}$

There were no significant associations found between SARSCoV-2 infection during pregnancy and adverse maternal or neonatal outcomes. This finding differs from other published studies showing possible associations between SARS-CoV-2 infection and preterm delivery, stillbirth, unplanned cesarean delivery, and/or preeclampsia., ${ }^{6,25-27}$ This difference is likely multifactorial. While 61 patients in our study population were infected with SARS-CoV-2 during their pregnancy, only two of these patients experienced severe disease requiring ICU admission. Only $27.9 \%$ experienced moderate-to-severe symptoms. A report of 241 births to women at five New York Medical centers during the initial surge of the pandemic showed increased rates for preterm birth and cesarean delivery but mostly among critically ill women. ${ }^{27}$ As the majority of our COVID-19-positive patients were not critically ill, their chance of having complications was likely lower.

Our seroprevalence rate is lower than pregnant populations in New York and Barcelona, likely reflecting an overall lower infection rate in a more rural population that is less likely to be exposed to and infected with the virus. However, our seroprevalence closely reflects that of the general 
population of Iowa at the time of this study. These rates are likely similar given that our institution serves a catchment area of nearly the entire state.

We did not collect specific information about why racial and ethnic minority groups may have been at higher risk of SARS-CoV-2 infection. However, many patients in the nonEnglish language and ethnic minority groups reported working in or living with family members who worked in high-risk occupations, such as meat processing plants, which had well-publicized outbreaks in our community during the study period. ${ }^{28}$ Patients in these ethnic and language groups are also more likely to work in jobs that are less likely to be possible to do from home. ${ }^{29}$

While not statistically significant, there was a notably higher percentage of COVID-19-positive than COVID-19-negative women with postpartum hemorrhage in the study (23.0 and $16.3 \%$, respectively). One other study by Liao et al found no significant difference in postpartum hemorrhage rates between SARS-CoV-2-positive and -negative mothers but their study population included only vaginal deliveries. ${ }^{30}$ This relationship warrants further study in a larger cohort of patients. Additionally, we found that COVID-19-positive patients were not statistically more likely to have a stillborn infant than COVID-19-negative mothers. There were only three stillborn infants born to COVID-19-positive patients in total: two due to IUFD and one who underwent termination of pregnancy due to significant fetal anomalies. Given that other studies have found increased rates of stillbirth in COVID-19-positive patients, this finding warrants additional study.

\section{Strengths and Limitations}

A strength of this study was that the 1,000 births recorded and analyzed were consecutive and represent all deliveries during the study period; no patients were excluded from the study analysis. SARS-CoV-2 RT-PCR results were electronically available for $98 \%$ (980) of study patients; the 20 patients without results either refused testing or delivered too quickly on admission to receive testing. These results allowed for comparison between SARS-CoV-2 viral and antibody testing.

A limitation of this study was the small nature of our total delivery cohort as compared with larger centers. Some results may have reached significance with a larger cohort. Additionally, there is evidence that access to SARS-CoV-2 RT-PCR testing was limited in our community early in the pandemic. Two of the patients who were SARS-CoV-2 viral negative and antibody positive reported COVID-19-like symptoms in March or April 2020 but did not have access to viral testing until admission for delivery.

\section{Conclusion}

This study adds to the limited body of literature on COVID-19 in pregnancy. In our largely White, rural, Midwestern population, SARS-CoV-2 infection was significantly higher in racial and ethnic minorities and immigrant populations, as well as patients without private insurance, suggesting a need for interventions to improve health equity in these groups. Our low disease severity and lack of maternal and infant outcomes related to SARSCoV-2 infection are encouraging but warrant further observation in the ongoing pandemic.

\section{Funding}

This study was funded internally. The study was also supported in part by the University of Iowa Institute for Clinical and Translational Science, which is granted with Clinical and Translational Science Award funds from the National Institutes of Health (UL1TR002537).

\section{Conflict of Interest}

None declared.

\section{Acknowledgments}

The authors acknowledge Ms. Ava Johnson and Ms. Laura Nicks for their contributions to the specimen collection for this manuscript. They are both employees of the University of Iowa and their work was funded internally.

\section{References}

1 COVID in Iowa. Positive case analysis Accessed November 3, 2020 at: https://coronavirus.iowa.gov/pages/case-counts

2 Qiao J. What are the risks of COVID-19 infection in pregnant women? Lancet 2020;395(10226):760-762

3 Schwartz DA, Graham AL. Potential maternal and infant outcomes from (Wuhan) coronavirus 2019-nCoV infecting pregnant women: lessons from SARS, MERS, and other human coronavirus infections. Viruses 2020;12(02):194

4 Ellington S, Strid P, Tong VT, et al. Characteristics of women of reproductive age with laboratory-confirmed SARS-CoV-2 infection by pregnancy status - United States, January 22-June 7, 2020. MMWR Morb Mortal Wkly Rep 2020;69(25):769-775

5 Akhtar H, Patel C, Abuelgasim E, Harky A. COVID-19 (SARS-CoV-2) infection in pregnancy: a systematic review. Gynecol Obstet Invest 2020;85(04):295-306

6 Narang K, Enninga EAL, Gunaratne MDSK, et al. SARS-CoV-2 infection and COVID-19 during pregnancy: a multidisciplinary review. Mayo Clin Proc 2020;95(08):1750-1765

7 Di Mascio D, Khalil A, Saccone G, et al. Outcome of coronavirus spectrum infections (SARS, MERS, COVID-19) during pregnancy: a systematic review and meta-analysis. Am J Obstet Gynecol MFM 2020;2(02):100107

8 Azar KMJ, Shen Z, Romanelli RJ, et al. Disparities in outcomes among COVID-19 patients in a large health care system in California. Health Aff (Millwood) 2020;39(07):1253-1262

9 Akbar AN, Gilroy DW. Aging immunity may exacerbate COVID-19. Science 2020;369(6501):256-257

10 Andrikopoulou M, Madden N, Wen T, et al. Symptoms and critical illness among obstetric patients with coronavirus disease 2019 (COVID-19) infection. Obstet Gynecol 2020;136(02):291-299

11 Savasi VM, Parisi F, Patanè L, et al. Clinical findings and disease severity in hospitalized pregnant women with coronavirus disease 2019 (COVID-19). Obstet Gynecol 2020;136(02):252-258

12 Merrill AE, Jackson JB, Ehlers A, Voss D, Krasowski MD. Head-tohead comparison of two SARS-CoV-2 serology assays. J Appl Lab Med 2020;5(06):1351-1357

13 Miller TE, Garcia Beltran WF, Bard AZ, et al. Clinical sensitivity and interpretation of PCR and serological COVID-19 diagnostics for patients presenting to the hospital. FASEB J 2020;34(10): 13877-13884

14 National Institutes of Health. Racial and ethnic categories and definitions for NIH diversity programs and for other reporting 
purposes. Accessed January 5, 2021 at: https://grants.nih.gov/ grants/guide/notice-files/not-od-15-089.html

15 Larson SB, Watson SN, Eberlein M, Simmons JS, Doerschug KC, Leslie KK. Survival of pregnant coronavirus patient on extracorporeal membrane oxygenation. Ann Thorac Surg 2020;S00034975(20):31634-31639

16 Haizler-Cohen L, Davidov A, Blitz MJ, Fruhman G. Severe acute respiratory syndrome corona virus 2 antibodies in pregnant women admitted to labor and delivery units. Am J Obstet Gynecol 2020;S0002-9378(20):31101-31107

17 Crovetto F, Crispi F, Llurba E, Figueras F, Gómez-Roig MD, Gratacós E. Seroprevalence and presentation of SARS-CoV-2 in pregnancy. Lancet 2020;396(10250):530-531

18 Sutton D, Fuchs K, D'Alton M, Goffman D. Universal screening for SARS-CoV-2 in women admitted for delivery. N Engl J Med 2020; 382(22):2163-2164

19 Miller ES, Grobman WA, Sakowicz A, Rosati J, Peaceman AM. Clinical implications of universal severe acute respiratory syndrome coronavirus 2 (SARS-CoV-2) testing in pregnancy. Obstet Gynecol 2020;136(02):232-234

20 Centers for Disease Control and Prevention. Births - method of delivery. Accessed September 2, 2020 at: https://www.cdc.gov/ nchs/fastats/delivery.htm

21 Iowa Public Health Tracking Portal. Adult obesity information. Accessed November 13, 2020 at: https://tracking.idph.iowa.gov/ Health/Obesity/Adult-Obesity-Data

22 Swank ML, Caughey AB, Farinelli CK, et al. The impact of change in pregnancy body mass index on the development of gestational hypertensive disorders. J Perinatol 2014;34(03):181-185
23 Price-Haywood EG, Burton J, Fort D, Seoane L. Hospitalization and mortality among black patients and white patients with COVID19. N Engl J Med 2020;382(26):2534-2543

24 Khalil A, Kalafat E, Benlioglu C, et al. SARS-CoV-2 infection in pregnancy: A systematic review and meta-analysis of clinical features and pregnancy outcomes. EClinicalMedicine 2020;25:100446

25 Kingston EV. High rates of stillbirth and preterm delivery in women with covid-19 and the efficacy of ECMO in pregnancy. BMJ 2020;370:m2921

26 Huntley BJF, Huntley ES, Di Mascio D, Chen T, Berghella V, Chauhan SP. Rates of maternal and perinatal mortality and vertical transmission in pregnancies complicated by severe acute respiratory syndrome coronavirus 2 (SARS-Co-V-2) infection: a systematic review. Obstet Gynecol 2020;136(02):303-312

27 Khoury R, Bernstein PS, Debolt C, et al. Characteristics and outcomes of 241 births to women with severe acute respiratory syndrome coronavirus 2 (SARS-CoV-2) infection at five New York city medical centers. Obstet Gynecol 2020;136(02):273-282

28 Leys T. Coronavirus infects more than 1,600 workers at four Iowa meatpacking plants. Accessed September 16, 2020 at: https:// www.desmoinesregister.com/story/news/health/2020/05/05/coronavirus-infects-thousands-iowa-meatpacking-plant-workerscovid-19-waterloo-perry/5170796002/

29 Dingel JI, Neiman B. How many jobs can be done at home? J Public Econ 2020;189:104235

30 Liao J, He X, Gong Q Yang L, Zhou C, Li J. Analysis of vaginal delivery outcomes among pregnant women in Wuhan, China during the COVID-19 pandemic. Int J Gynaecol Obstet 2020;150 (01):53-57 